ARTICLE

Received 12 Sep 2013 | Accepted 30 Apr 2014 | Published 5 Jun 2014 DOl: 10.1038/ncomms5013

\title{
Design amphiphilic dipolar $\pi$-systems for stimuli-responsive luminescent materials using metastable states
}

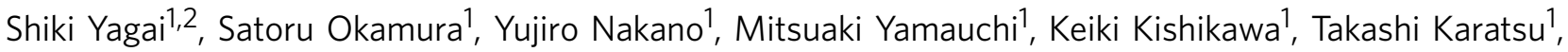
Akihide Kitamura', Akira Ueno ${ }^{3}$, Daiki Kuzuhara ${ }^{4}$, Hiroko Yamada ${ }^{2,4}$, Tomohiro Seki ${ }^{5}$ \& Hajime Ito $^{5}$

$\pi$-Conjugated compounds that exhibit tunable luminescence in the solid state under external mechanical stimuli have potential applications in sensors and imaging devices. However, no rational designs have been proposed that impart these mechano-responsive luminescent properties to $\pi$-conjugated compounds. Here we demonstrate a strategy for mechanoresponsive luminescent materials by imparting amphiphilic and dipolar characteristics to a luminescent $\pi$-conjugated system. The oligo( $p$-phenylenevinylene) luminophore with a didodecylamino group at one end and a tri(ethylene glycol) ester group at the other end yields segregated solid structures by separately aggregating its hydrophobic and hydrophilic moieties. The segregated structures force the molecules to align in the same direction, thereby generating a conflict between the side-chain aggregation and dipolar stabilization of the $\pi$-system. Consequently, these metastable solid structures can be transformed through mechanical stimulation to a more stable structure, from a $\pi-\pi$ stacked aggregate to a liquid crystal and further to a crystalline phase with variable luminescence.

\footnotetext{
${ }^{1}$ Graduate School of Engineering, Chiba University, 1-33 Yayoi-cho, Inage-ku, Chiba 263-8522, Japan. 2 Japan Science and Technology Agency (JST), Core Research for Evolutional Science and Technology (CREST), Chiyoda-ku, Tokyo 102-0076, Japan. ${ }^{3}$ Microjet Corporation, 5-79-2 Daimon, Shiojiri, Nagano 399-0732, Japan. ${ }^{4}$ Graduate School of Material Science, Nara Institute of Science and Technology (NAIST), 8916-5, Takayama-cho, Ikoma, Nara 630-0192, Japan. ${ }^{5}$ Division of Chemical Process Engineering and Frontier Chemistry Center (FCC), Graduate School of Engineering, Hokkaido University, Kita 13 Nishi 8 , Kita-ku, Sapporo 060-0810, Japan. Correspondence and requests for materials should be addressed to S.Y. (email: yagai@faculty.chiba-u.jp).
} 
$\mathrm{O}$ rganic, $\pi$-conjugated materials that exhibit tunable luminescent properties in the solid state under external stimuli can be applied in optical devices, displays, fluorescent probes and sensors ${ }^{1-4}$. Recently, luminescent materials that are responsive to mechanical stimuli have attracted considerable attention because they have the unique ability to tune their molecular, physical or chemical properties via macroscopic stimulation ${ }^{5-8}$. Several mechanistic explanations for the mechanically stimulated changes in the colour of the luminescence have been proposed: a phase transformation of the solid structure ${ }^{9-16}$, excimer formation or dissolution ${ }^{17-19}$ and mechanical stress-induced chemical changes ${ }^{20,21}$. However, the development of new materials with luminescent mechanochromism still depends heavily on serendipitous discovery or random screening; studies based on rationally designed luminophoric compounds and sensitivity to mechanical stimuli remains rare. One possible strategy for designing stimulus-sensitive materials involves generating metastable, kinetically trapped states in the luminophoric compounds with emission properties that are sensitive to the surrounding environment. When a mechanical stimulus converts the metastable state to a more thermodynamically stable solid state with new emission properties, a mechano-responsive change occurs in the compound's luminescence.

Here we report a new mechanochromic material created using a molecular-level design according to the aforementioned strategy. To obtain metastable, self-assembled states, we imparted amphiphilic and dipolar characteristics to a luminescent $\pi$-conjugated system. The $\pi$-conjugated molecule can adopt a segregated solid structure by aggregating its hydrophobic and hydrophilic moieties separately (Fig. 1a) ${ }^{22-24}$. The segregated structure would force the molecules to align in the same direction, thereby generating a conflict between the side-chain aggregation and dipolar stabilization of the core ${ }^{22}$. Consequently, this designed metastable aggregate structure could be easily transformed to more stable structures with variable luminescence.

\section{Results}

Molecular design. In this study, molecule $\mathbf{1}$ was designed to exhibit various stimuli-responsive properties (Fig. 1b). Compound 1 contains three different segments: a polarized luminophoric core of oligo( $p$-phenylenevinylene $)^{25-27}$, an electron-donating amino group with hydrophobic dodecyl chains $\left(\mathrm{C}_{12} \mathrm{H}_{25}\right)$ and an electron-withdrawing ester group with a hydrophilic tri(ethylene glycol) (TEG) chain. The amino and ester groups render the core portion moderately polar $(\mu=6.8 \mathrm{D}$ based on a density functional theory (DFT) calculation) and impart intramolecular charge-transfer abilities to the excitation state because the destabilized highest occupied molecular orbital (HOMO) is more localized around the amino group, whereas the stabilized lowest unoccupied molecular orbital (LUMO) remains more localized around the ester group (Fig. 2a). In solution, 1 exhibits a notable solvatochromic photoluminescence shift from blue to red when the solvent polarity changes from $\varepsilon$ (dielectric constant $)=1.88 \quad$ (hexane, $\left.\lambda_{\text {em-max }}=488 \mathrm{~nm}, \Phi_{\mathrm{F}}=0.79\right) \quad$ to $\varepsilon=37.5 \quad$ (acetonitrile, $\lambda_{\mathrm{em}-\max }=685 \mathrm{~nm}, \Phi_{\mathrm{F}}=0.23$ ) (Fig. 2b), whereas the absorption spectra remain unchanged (Supplementary Fig. 1 and Supplementary Table 1). These results indicate that $\mathbf{1}$ has excited-state energy levels associated with intramolecular charge transfers. Therefore, in the condensed state, the photoemission properties of 1 should also be sensitive to the microenvironment and to molecular arrangements.

Mechanochromic luminescence. Compound 1 initially exhibited yellow photoluminescence after being drop cast onto a glass substrate; after the sample was pressed, the luminescence became orange. An anisotropic mechanical stimulus (rubbing) applied to
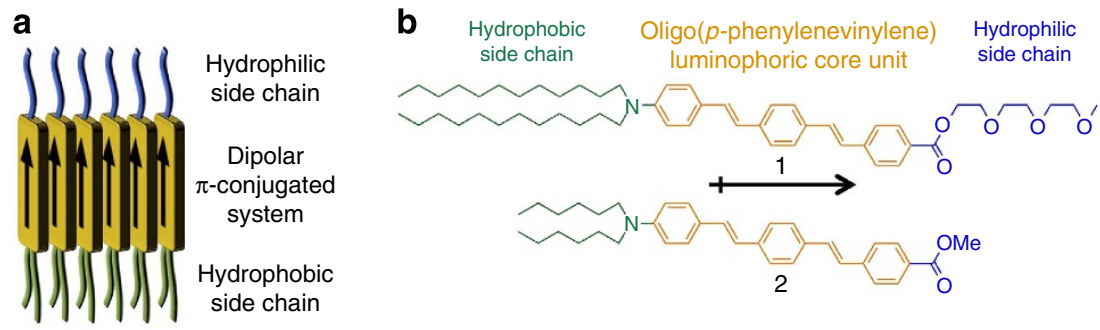

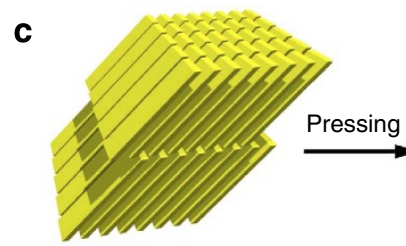

Aggregate

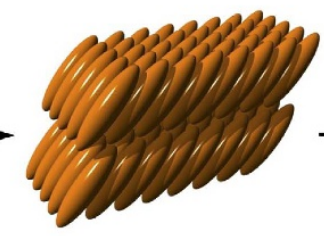

Liquid cystal

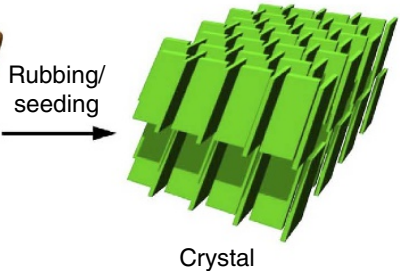

d
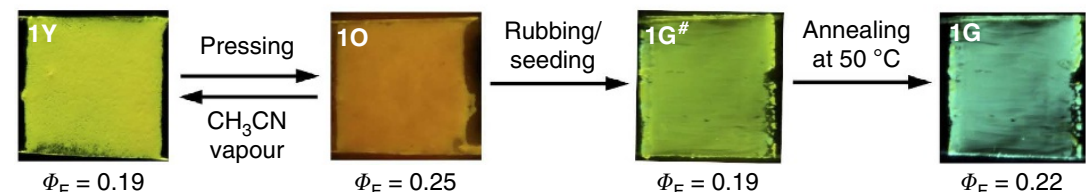

Figure 1 | Stimuli-responsive change in luminescence and phase of 1. (a) Schematic representation of the segregated packing structure of amphiphilic dipolar molecule. (b) Molecular structure of amphiphilic dipolar luminophore $\mathbf{1}$ and reference compound $\mathbf{2}$. (c) Schematic representation of stimuli-

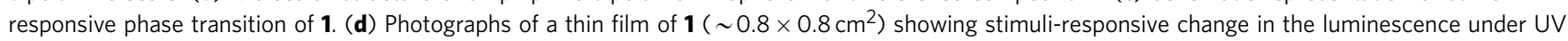
$(365 \mathrm{~nm})$ irradiation. 


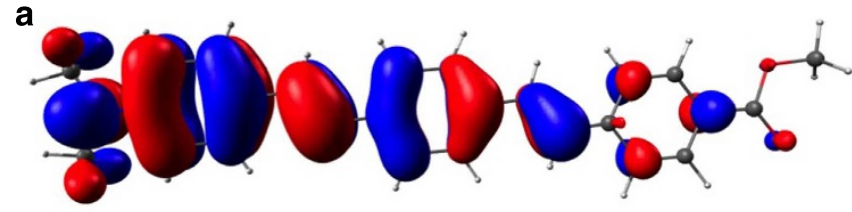

HOMO

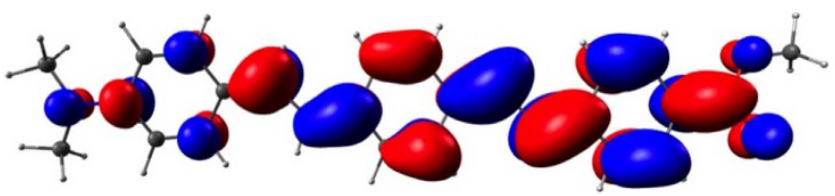

LUMO

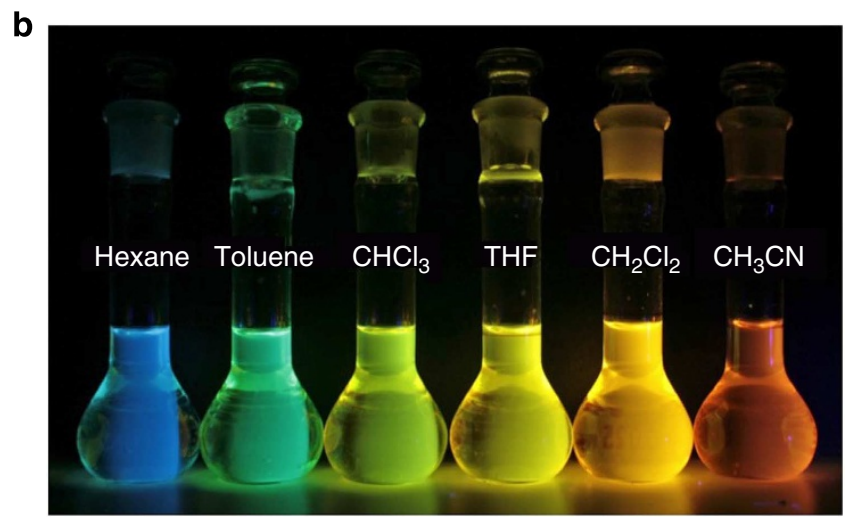

Figure 2 | Molecular orbitals and photoluminescence colors of dipolar oligo(p-phenylenevinylene) in various solvents. (a) Molecular orbitals (top: HOMO and bottom: LUMO) of a model dipolar oligo( $p$ phenylenevinylene) molecule (asymmetrically substituted with $-\mathrm{COOCH}_{3}$ and - $\mathrm{N}\left(\mathrm{CH}_{3}\right)_{2}$ groups) obtained by DFT calculation at B3LYP/6-

$311++\mathrm{G}(\mathrm{d}, \mathrm{p})$. (b) Photographs of the solutions of $\mathbf{1}$ in various organic solvents (from left to right: hexane, toluene, chloroform, tetrahydrofuran, dichloromethane and acetonitrile) under UV (365 nm) irradiation.

the material's surface triggered a phase change to a crystalline material with green photoluminescence. Even when the rubbing area was small, the phase conversion spontaneously propagated across the entire sample area. An analysis of the structural and optical properties of the phases revealed that the variable luminescence originates from the alteration of the intermolecular interaction and the conformation of the $\pi$-conjugated luminophores upon the mechanical induction of phase transitions from aggregate to mesophase to crystal (Fig. 1c).

Figure 1d outlines the stimuli-responsive luminescent colour change of 1, whereas Fig. 3 illustrates optical microscopy (OM), polarized $\mathrm{OM}$ (POM) and fluorescence microscopy images typical of each phase. We obtained a thin film (1Y) that exhibited yellow photoluminescence (fluorescence quantum yield, $\left.\Phi_{\mathrm{F}}=0.19\right)$ after casting aqueous acetonitrile solutions of 1 . OM and POM images showed the formation of birefringent, sheet-like micro objects (microsheets; Fig. 3a,b). After being pressed or gently ground, $1 Y$ became a waxy, fluidic and highly birefringent mesophase (10; Fig. $3 \mathrm{c}, \mathrm{d}$ ). The $\mathbf{1 Y} \rightarrow \mathbf{1 0}$ phase transition was accompanied by a change in the photoluminescence from yellow to orange $\left(\Phi_{\mathrm{F}}=0.25\right)$ (Supplementary Movie 1$)$. The mesomorphic phase 10 could be reverted to $\mathbf{1 Y}$ by exposure to acetonitrile vapour. To our surprise, vigorous grinding of $\mathbf{1 0}$ triggered a crystallization and a further change in the photoluminescence to yellowish green $\left(\mathbf{1 G}^{\#}, \Phi_{\mathrm{F}}=0.19\right)$. Thermal annealing of $\mathbf{1 G}^{\#}$ at $50^{\circ} \mathrm{C}$ generated a bluish-green emission
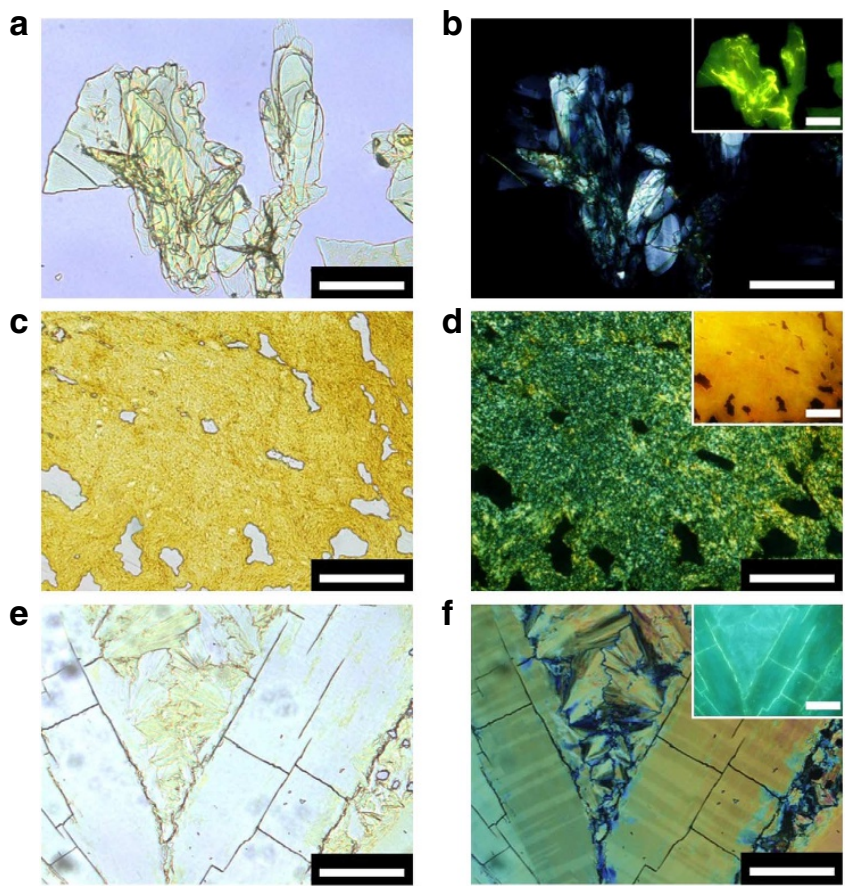

Figure 3 | Optical micrographs showing mechanically induced phase transition of 1. (a,b) Optical micrograph (a) and polarized optical micrograph (b) of microsheets of $\mathbf{1 Y}$. (c,d) Optical micrograph (c) and polarized optical micrograph (d) of mesophase of 10. (e,f) Optical micrograph (e) and polarized optical micrograph (f) of crystallites of $\mathbf{1 G}$ Scale bars, $100 \mu \mathrm{m}$ in all the images. Insets in $\mathbf{b}, \mathbf{d}$ and $\mathbf{f}$ are the corresponding fluorescence micrographs of each phase.

(1G, $\Phi_{\mathrm{F}}=0.22$; Fig. 3e,f). Because POM (Supplementary Fig. 2a), differential scanning calorimetry (DSC; Supplementary Fig. 2b), powder X-ray diffraction (PXRD; Supplementary Fig. 3) and ultraviolet/visible (UV/Vis) studies (Supplementary Fig. 4) indicated that $\mathbf{1 G}^{\#}$ was a less crystalline form of $\mathbf{1 G}$, we focused on 1Y, 10 and 1G during the subsequent structural studies.

Thermal properties. The DSC analyses revealed that $1 Y, 10$ and $1 G$ exhibit different thermal stabilities (Fig. 4). A heated scan of $1 Y$ revealed a broad endothermic peak at $\sim 37^{\circ} \mathrm{C}(\Delta H=11.5$ $\left.\mathrm{kcal} \mathrm{mol}^{-1}\right)$. POM observations showed that this peak was caused by the thermal transition of $\mathbf{1 Y}$ to $\mathbf{1 O}$ (see below). The resultant 10 became the isotropic phase (Iso) at $47^{\circ} \mathrm{C}\left(\Delta H=1.4 \mathrm{kcal} \mathrm{mol}^{-}\right.$ $\left.{ }^{1}\right)$; this result is consistent with the clearing temperature of the mechanically induced $10\left(47^{\circ} \mathrm{C}, \Delta H=4.4 \mathrm{kcal} \mathrm{mol}^{-1}\right)$. These results indicate that the molecular packings of mechanically and thermally induced $\mathbf{1 0}$ are potentially identical. In contrast, the crystalline phase $1 \mathrm{G}$ transformed into Iso at $54^{\circ} \mathrm{C}(\Delta H=12.7$ $\left.\mathrm{kcal} \mathrm{mol}^{-1}\right)$, achieving the most stable molecular packing. All isotropic phases exhibited two exothermic transitions at $\sim 44^{\circ} \mathrm{C}$ and $38^{\circ} \mathrm{C}$, respectively, upon cooling, without the hysteresis observed in the pre-melt phases. The POM observations indicated that the upper transition corresponded to the thermal generation of mesomorphic 10, whereas the lower transition was accompanied by no apparent change in the POM textures as well as emission properties. Taking into account a small change of interlayer distance $(d=32.6 \rightarrow 34.2 \AA$, see the next section $)$ and a small enthalpy change $\left(-0.5 \mathrm{kcal} \mathrm{mol}^{-1}\right)$ upon the lower transition, a minor structural change that is associated with the molecular tilt might occur in $\mathbf{1 0}$ at $38^{\circ} \mathrm{C}$ upon cooling.

Molecular packing. The PXRD measurements of 1's three distinct phases indicated that the mechanical stimuli induced explicit 

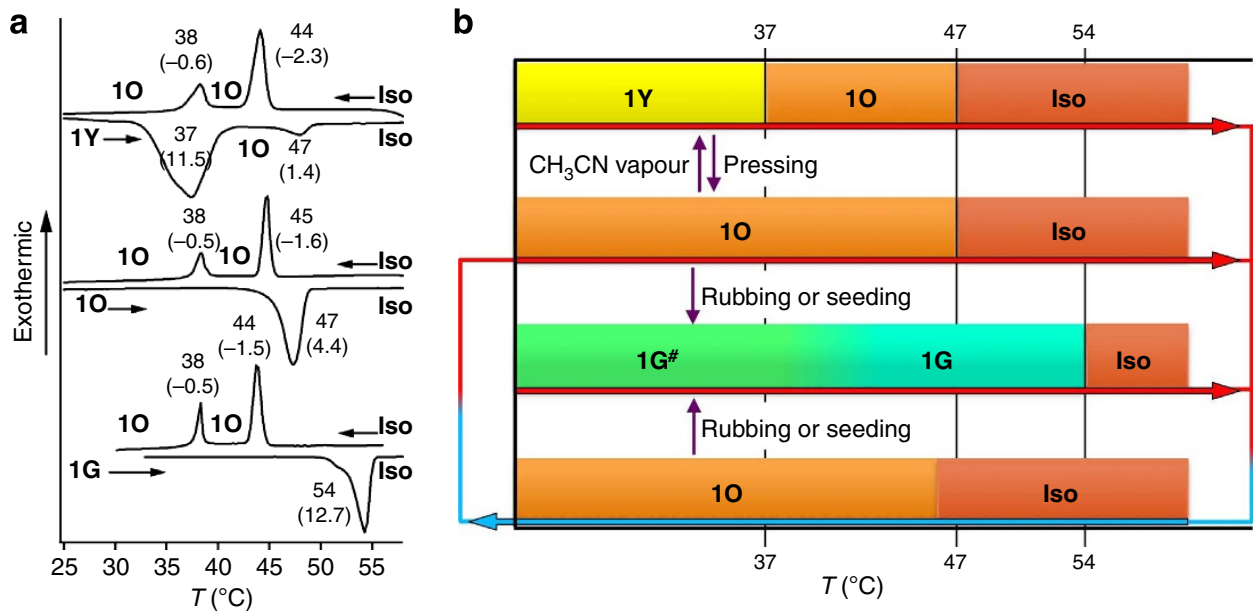

Figure 4 | Thermal behaviour of 1. (a) DSC traces $\left(2{ }^{\circ} \mathrm{C} \min ^{-1}\right.$ ) of $\mathbf{1 Y}, \mathbf{1 0}$ and $\mathbf{1 G}$ with the phase-transition temperatures in ${ }^{\circ} \mathrm{C}$ and associated enthalpy changes (in brackets) in $\mathrm{kcal} \mathrm{mol}^{-1}$. (b) Phase-transition behaviour of $\mathbf{1}$. Heating and cooling procedures are indicated by red and blue arrows, respectively.

a

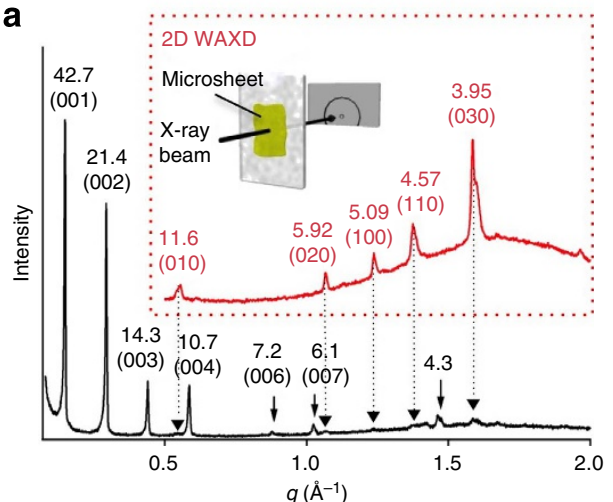

e

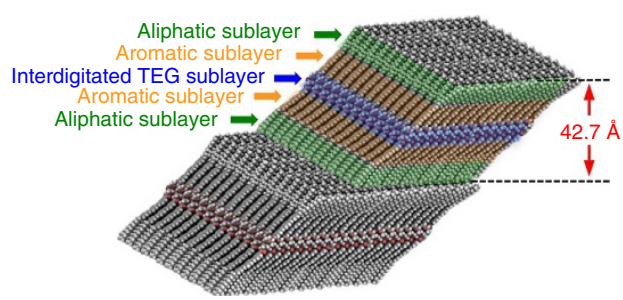

b
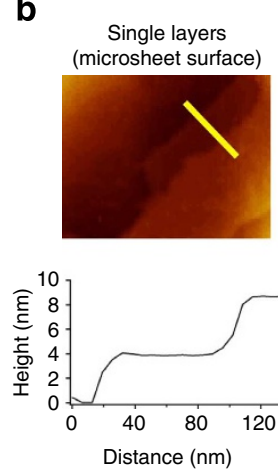

C

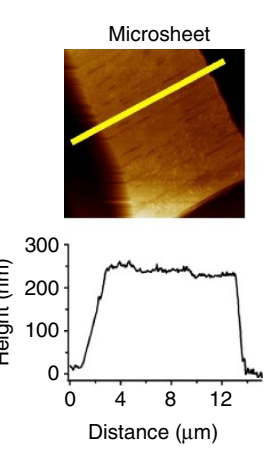

d

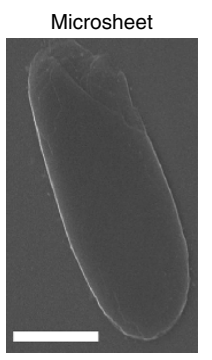

$\mathbf{f}$

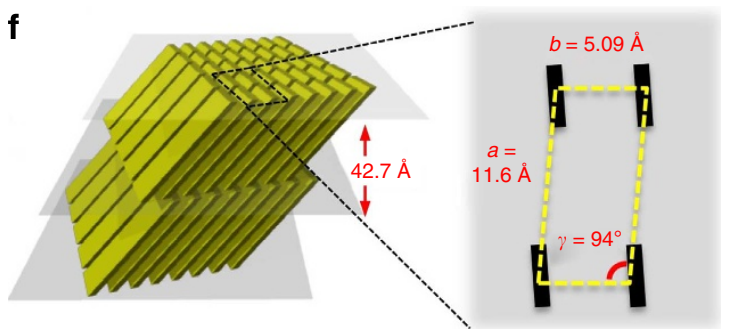

Figure 5 | Analysis of packing structures of 1Y. (a) PXRD patterns of $\mathbf{1 Y}$ with major $d$-spacings in $\AA$. Small diffractions derived from the in-plane molecular ordering are indicated by black triangles. Inset (surrounded by red dotted square): Schematic representation for the experimental setup of transmission mode 2D-WAXD of $\mathbf{Y Y}$ microsheets deposited on 0.03- $\mathrm{mm}$ thickness glass substrate using synchrotron radiation and the observed diffraction profile. (b) AFM image of the surface of $\mathbf{1 Y}$ microsheet and a cross-sectional analysis along to the yellow line. (c) AFM image of the surface of $\mathbf{1 Y}$ microsheet and a cross-sectional analysis along to the yellow line. (d) SEM image of a $\mathbf{1 Y}$ microsheet (scale bar, $10 \mu \mathrm{m}$ ). (e) Schematic representation of the multilamellar structure of $\mathbf{1 Y}$. Aromatic, aliphatic and hydrophilic sublayers are indicated by orange, green and yellow, respectively. (f) Schematic representation of in-plane oblique packing of $\mathbf{1}$ in $\mathbf{1 Y}$. The yellow plates correspond to two molecules of $\mathbf{1}$.

transformations in the material's molecular packing. The PXRD pattern of $\mathbf{1 Y}$ was characterized by numerous reflections with a reciprocal spacing ratio $1: 2: 3: 4: 6: 7$ for $42.7 \AA$ (Fig. 5a). Atomic force microscopy (AFM) observation of the surface of the microsheets of $1 Y$ visualized the elementary layers with thicknesses of $\sim 4 \mathrm{~nm}$ (Fig. 5b), which is consistent with the PXRD results. The emergence of up to (007) reflections demonstrates that a long-range ordered, multilamellar structure was formed. Because the molecular length of 1 was $\sim 42 \AA$, the observed layer spacing indicates that a bilayer structure was formed with a considerable molecular tilt and interdigitated TEG side chains
(Fig. 5e). In the wide-angle region, the reflection corresponding to the crystalline alkyl chain was observed at $d=4.3 \AA$, along with other small reflections that could be derived from the in-plane molecular ordering within the lamellae.

The in-plane molecular ordering in the $\mathbf{1 Y}$ microsheet was further revealed by two-dimensional (2D) wide-angle X-ray diffraction (WAXD) analysis. AFM (Fig. 5c) and scanning electron microscopy (SEM; Fig. 5d) images showed that the microsheets of $1 Y$ have thicknesses of $100-250 \mathrm{~nm}$ and were formed in the face-on arrangement on the glass substrate. Transmission mode 2D WAXD analysis using the X-ray beam 
perpendicular to the microsheets showed diffractions corresponding to $d$-spacings of $11.6,5.92,5.09,4.57$ and $3.95 \AA$ (inset in Fig. 5a). These diffractions were assigned to an oblique lattice with lattice parameters $a=11.6 \AA, b=5.09 \AA$ and $\gamma=94^{\circ}$ (Fig. 5f). The short lattice parameter $b$ indicates the presence of cofacially $\pi$ - $\pi$-stacked $\pi$-conjugated moieties.

In contrast, the PXRD patterns of $\mathbf{1 0}$ were fairly simple and were attributed to a lamellar mesophase with a $34.2-\AA$ interlayer spacing (Fig. 6a). The absence of reflections for in-plane ordering may reflect the dynamic nature of the individual molecules in this phase; the $\pi$-conjugated chromophores were rotating around their long molecular axis. When $\mathbf{1 0}$ was thermally generated on a glass substrate via slow cooling of the isotropic melt, a four-brush schlieren texture was observed by POM (Fig. 6b). For thinner 10 samples prepared by sandwiching two glass substrates, a focalconic fan texture was observed with extinction brushes that were inclined by $45^{\circ}$ with respect to the crossed polarizers (Fig. 6c). These POM textures are typical of smectic C liquid crystalline mesophases with homeotropic (schlieren) and planar orientations (focal-conic fan). The 2D WAXD pattern of sheared 10 showed no diffraction peaks in the small-angle region, suggesting the absence of in-plane molecular ordering.

The crystalline phase $\mathbf{1 G}$ exhibited the most complicated diffraction pattern that contained an intense diffraction at $38.6 \AA$ and a number of weak diffractions indicative of crystallizationinduced, 3D molecular order (Fig. 6d). We found that this crystalline phase became waxy when heated in the range of 40$50{ }^{\circ} \mathrm{C}$, whereas the emission colour remained green. This behaviour is possibly because of the melting of the TEG sublayers that can be used to orient the molecules mechanically. Therefore, we performed an in-plane, 2D PXRD measurement of a thin-film sample of 1G sheared on a glass substrate at $42{ }^{\circ} \mathrm{C}$ (Fig. 6e). On the meridian, a diffraction corresponding to the interlayer distance was observed (Fig. 6f), which suggested that the basic lamellar structure would be preserved upon crystallization of 10 to $1 \mathrm{G}$. In the wide-angle region, broad diffractions were observed at 4.8, 3.9 and $3.6 \AA$; the centres of these diffraction peaks were not located on the meridian (Fig. 6g). Although a clear assignment of the diffraction pattern could not be made, these observations suggest that the inclination of the molecules within the layers is also preserved upon crystallization of $\mathbf{1 0}$.

The parent compound $\mathbf{1}$ did not form crystals suitable for $\mathrm{X}$-ray crystallography; however, we obtained structural insight into the mesomorphic $\mathbf{1 0}$ from a single-crystal structural analysis of a reference compound (2) that lacked amphiphilic side chains (Supplementary Data 1). Compound 2 yielded plate-like single crystals from an ethyl acetate solution that exhibited an orange emission (Supplementary Fig. 5a). Because the emission spectrum of crystal $\mathbf{2}$ is very similar to that of $\mathbf{1 0}$ (Fig. 7a), the crystal structural analysis of 2 revealed the packing state of the $\pi$-conjugated core in the mesomorphic phase 10. The crystal structure of 2 has a triclinic space group $P-1$ and is characterized by a lamellar ordering of an inclined molecular packing (Supplementary Fig. 5b). The dipolar molecules are packed within single layers in the antiparallel arrangement because of the absence of amphiphilicity. When the lamellar structure is viewed from the direction of the molecular long axis, a herringbone arrangement of the $\pi$-conjugated planes is revealed (Fig. 7b). The $\pi$-conjugated planes between the neighbouring herringbone rows have a dihedral angle of $40.4^{\circ}$, suggesting the absence of $\pi-\pi$ stacking interactions. This finding indicates that the orange emission is derived from the oligo( $p$-phenylenevinylene) luminophores with no apparent overlap of their $\pi$-planes. More importantly, the oligo( $p$-phenylenevinylene) cores alternately adopt chair-like or boat-like conformations from row to row (Fig. 7b). Whereas the chair-like conformer is ordered, the boatlike conformer is disordered because of the adoption of two crystallographically independent conformations with different occupancies (I:II = 41:59; Fig. $7 \mathrm{c})^{28}$. The temperature-dependent crystallographic analyses performed at temperatures from -150 to $20^{\circ} \mathrm{C}$ did not show a change in the occupancies of the two boat-like conformers, which suggests that they are not dynamically interconverted in the crystal. Nevertheless, the boatlike conformers I and II exhibit a geometrical distortion: the two $\mathrm{C}-\mathrm{C}$ bonds linking the central phenylene unit and the vinylene groups show an angle $(\theta)$ of $161^{\circ}$ and $167^{\circ}$, respectively, which deviate from linearity compared with the nearly linear shape of the chair-like conformer $\left(\theta=178^{\circ}\right.$; Fig. $\left.7 \mathrm{~d}\right)$. This geometrical
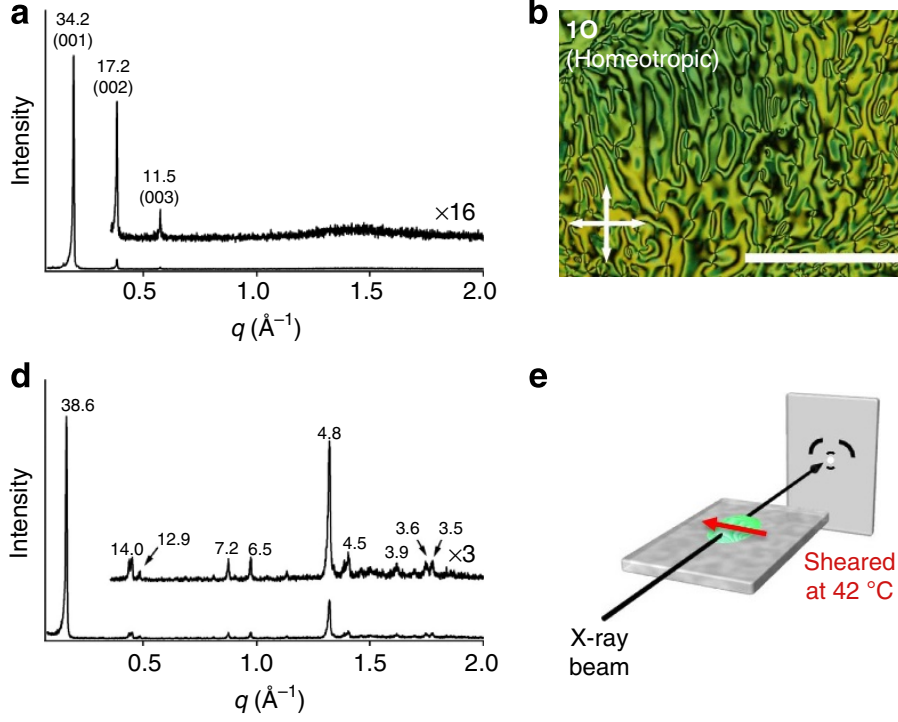
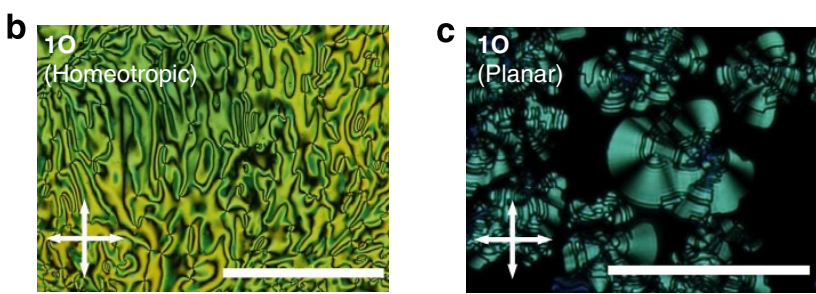

f

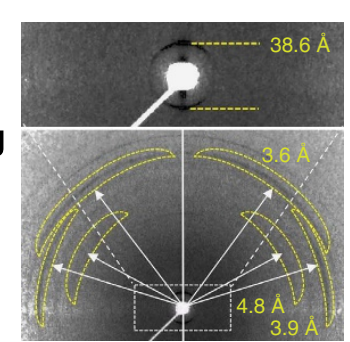

Figure 6 | Analysis of packing structures of $\mathbf{1 0}$ and 1G. (a) PXRD pattern of 10. (b,c) Polarized optical micrographs of homeotropically oriented $\mathbf{1 0}$ on a glass substrate (b, scale bar, $100 \mu \mathrm{m}$ ) and planarly oriented $\mathbf{1 0}$ between two glass substrates (c, scale bar, $20 \mu \mathrm{m}$ ). The samples were prepared via thermal procedure. Arrows indicate the direction of polarizer and analyser. (d) PXRD pattern of $\mathbf{1 G}$. (e) Schematic representation for the experimental setup of in-plane 2D PXRD measurements of $\mathbf{1 G}$ sheared at $42{ }^{\circ} \mathrm{C}$ along to the red arrow. $(\mathbf{f}, \mathbf{g}) 2 \mathrm{D}$ diffraction pattern of the sheared $\mathbf{1 G}$ in small-angle (f) and wide-angle region ( $\mathbf{g}$ ). For $\mathbf{g}$, broad diffractions were surrounded by yellow dotted lines. 

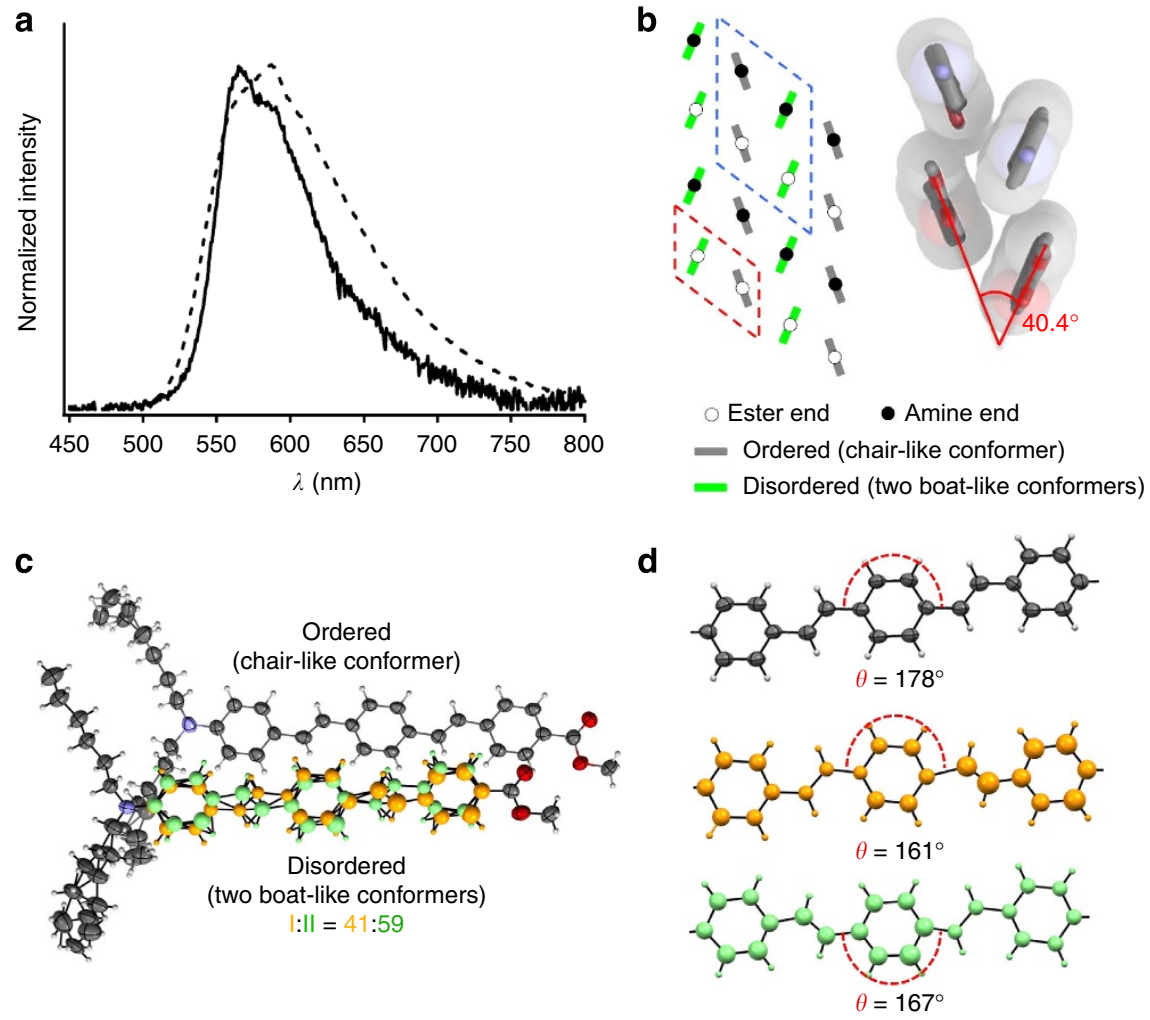

Figure 7 | X-ray crystallographic study of 2. (a) Fluorescence spectra $\left(\lambda_{\mathrm{ex}}=400 \mathrm{~nm}\right.$ ) of $\mathbf{2}$ (solid line) and $\mathbf{1 0}$ (dashed line). (b) Left: schematic representation of the herringbone arrangement of the oligo( $p$-phenylenevinylene) $\pi$-conjugated unit by viewing from the direction along to the long molecular axis. Grey and green bars indicate the ordered and disordered $\pi$-conjugated units, respectively. Open and closed circles indicate the ester and amine end of the $\pi$-conjugated units, respectively. Right: the molecular packing of a tetrameric unit extracted from the crystal structure (surrounded by blue dotted lines). (c) ORTEP drawing of a dimeric unit (surrounded by red dotted lines in $\mathbf{b}$ ) composed of the crystallographically ordered $\pi$-conjugated unit with a chair-like conformation (grey) and crystallographycally disordered $\pi$-conjugated units with two boat-like conformations. Conformers I and II are coloured with yellow and green, respectively. Occupancies of the conformers I and II are $41 \%$ and 59\%, respectively.

(d) Comparison of the distortion of $\pi$-conjugated systems in the three conformers.

distortion must be responsible for the long-wavelength emission because of intramolecular charge-transfer interactions.

Optical properties. Solid-state absorption measurements revealed that only $1 Y$ featured a strong exciton interaction between the $\pi$-conjugated luminophores (left axis; Fig. 8a). A solution of monomeric $\mathbf{1}$ in acetonitrile exhibited an intense absorption band at $412 \mathrm{~nm}$. After the solution was cast to form 1Y films, a large hypsochromic shift to $333 \mathrm{~nm}$ was observed. According to the exciton theory ${ }^{29}$, this spectral change is indicative of $\pi$-conjugated chromophores stacked face-to-face (H-type aggregation). When $\mathbf{1 Y}$ was converted to $\mathbf{1 0}$ by gentle pressing, the absorption band at $390 \mathrm{~nm}$ increased, reaching almost the same intensity as the hypsochromically shifted band and clearly demonstrating a decrease in the electronic interactions between the $\pi$-conjugated chromophores in the mesomorphic state. The spectrum of $\mathbf{1 G}$ resembled that of $\mathbf{1 0}$ but was more vibronically structured to reflect the rigid molecular packing in this phase. These absorption features, combined with the DSC and PXRD results, demonstrate that the mechanochromic luminescence from $\mathbf{1 Y}$ to $\mathbf{1 O}$ was caused by the loss of excitonic interactions between the dipolar $\pi$-conjugated systems, whereas that from $\mathbf{1 0}$ to $\mathbf{1 G}$ was caused by the increased rigidity of the molecular structure due to crystallization.

The emission properties of the three distinct phases were studied via steady-state (right axis; Fig. 8a) and time-resolved fluorescence spectroscopy (Fig. 8b), and the fluorescence quantum yield $\left(\Phi_{\mathrm{F}}\right)$, average fluorescence lifetime $\left(\tau_{\mathrm{av}}\right)$ and radiative rate constant $\left(k_{\mathrm{F}}\right)$ are summarized in Supplementary Table 2. We also measured the fluorescence properties of 1 dissolved in various solvents with different polarities (Supplementary Table 1), and compared the values with those of the solid phase by plotting the results against the emission maxima (Fig. 8c-e). All the solid phases displayed smaller $\Phi_{\mathrm{F}}$ (Fig. 8c) and $k_{\mathrm{F}}$ (Fig. 8e) values compared with those in the solution phases; these smaller values can be attributed to possible energy transfer in the bulk materials. In contrast to the nearly $\lambda_{\text {em-max }}$-independence of $\tau_{\mathrm{av}}(1-2 \mathrm{~ns})$ in the solution phases, the $\tau_{\mathrm{av}}$ of the solid phases exhibited a large dependence on $\lambda_{\mathrm{em} \text {-max }}$ (Fig. 8d). This observation suggests that the geometrical freedom and/or intermolecular interaction of luminophores are responsible for the emission wavelength in the solid states.

The $\mathrm{H}$-aggregated phase $\mathbf{1 Y}$ had a maximum emission at $545 \mathrm{~nm}$ with a 3.24-ns lifetime and a radiative rate constant of $5.9 \times 10^{7} \mathrm{~s}^{-1}$. In general, H-type aggregation causes strong fluorescence quenching, as is typically observed for dipolar merocyanine dyes ${ }^{30,31}$. If the largest Stokes shift $\left(11,681 \mathrm{~cm}^{-1}\right)$ among the three phases is taken into consideration, the fluorescence from 1Y could arise from an imperfect H-type stacking with a rotational displacement around the stacking axis, thereby allowing the radiative decay to originate from the lower-lying exciton state ${ }^{32}$.

The mesomorphic phase $\mathbf{1 0}$ exhibited a more red-shifted emission that reached a maximum at $587 \mathrm{~nm}$ (Stokes shift $=$ $8,539 \mathrm{~cm}^{-1}$ ) and was unchanged after transformation into isotropic phase Iso. The longest $\tau_{\mathrm{av}}$ (Fig. $8 \mathrm{~d}$ ) but the same 

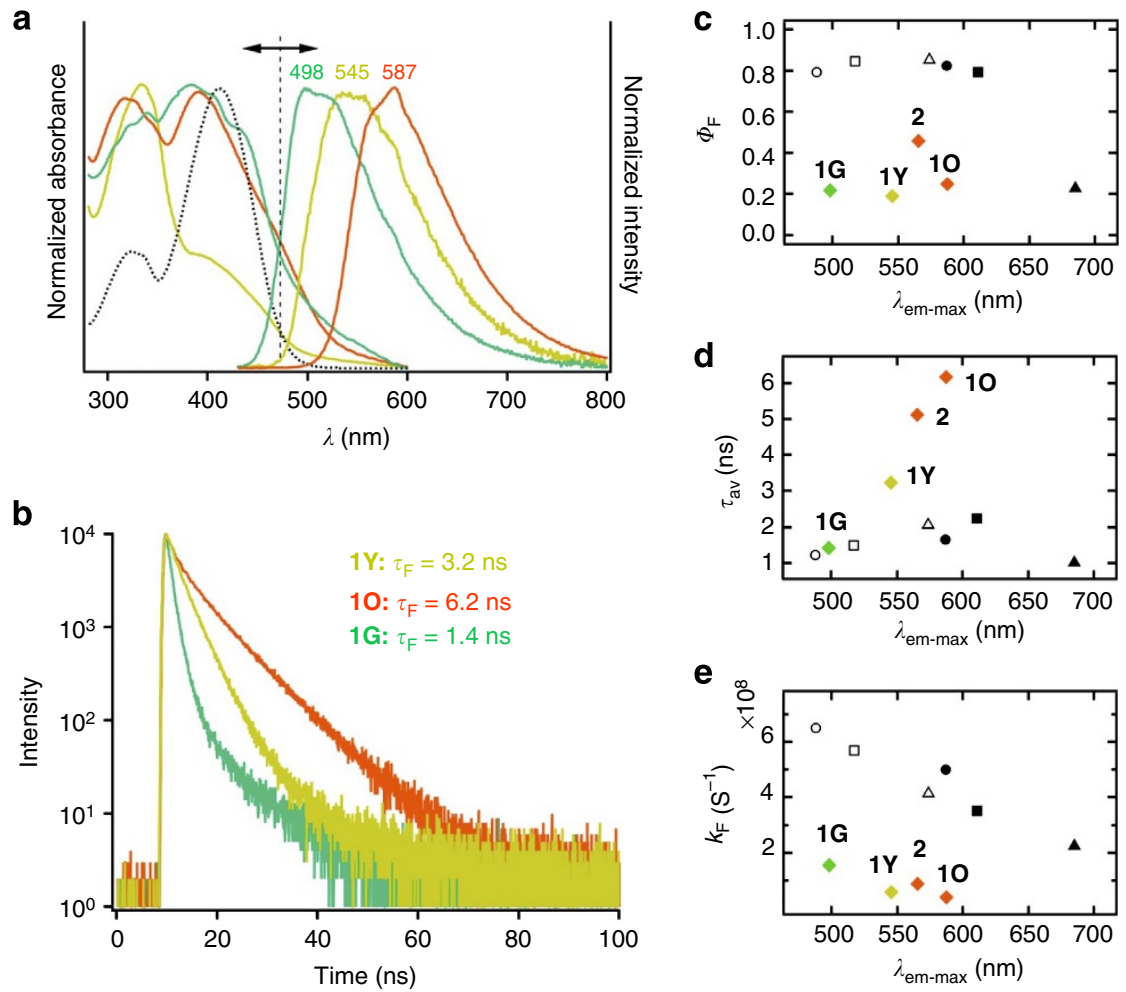

Figure 8 | Absorption and fluorescence properties of 1. (a) UV/Vis absorption (left axis) and fluorescence spectra (right axis, $\lambda_{\mathrm{ex}}=400 \mathrm{~nm}$ ) of 1Y, 10 and 1G. The dotted curve represents the UV/Vis spectrum of the acetonitrile solution $\left(c=1.0 \times 10^{-5} \mathrm{M}\right)$. (b) Fluorescence decay profiles of $\mathbf{1 Y}, \mathbf{1 0}$ and $\mathbf{1 G}$ with their fluorescence lifetimes $\left(\tau_{\mathrm{F}}\right)$. (c-e) Scatter plot of fluorescence quantum yield (c), average fluorescence lifetime (d) and radiative rate constant (e) of $\mathbf{1 G}, \mathbf{1 Y}, \mathbf{1 0}$, single crystal of $\mathbf{2}$ and solutions of $\mathbf{1}$ (open circles, hexane; open squares, toluene; open triangles, chloroform; closed circles, tetrahydrofuran; closed squares, dichloromethane; and closed triangles, acetonitrile).

approximately $k_{\mathrm{F}}$ value as that of $\mathbf{1 Y}$ (Fig. 8e) indicates that the intermolecular energy transfer was suppressed in $\mathbf{1 0}$ compared with in $1 Y$ because of the absence of the excitonic interaction between the luminophores. Importantly, the crystal of $\mathbf{2}$ involving distorted $\pi$-conjugated moieties exhibited an orange fluorescence with emission properties analogous to those of 10 (Fig. 8d,e), except for a higher $\Phi_{\mathrm{F}}(0.46$; Fig. $8 \mathrm{c})$. Therefore, similar geometric distortion of the $\pi$-conjugated systems occurs in this fluid phase's excited state, which may facilitate intramolecular charge-transfer interactions to generate a relatively long-lived, red-shifted emission.

In contrast, the crystalline phase $\mathbf{1 G}$ exhibited an emission maximum at the shortest wavelength $(498 \mathrm{~nm})$ with the smallest Stokes shift $\left(6,090 \mathrm{~cm}^{-1}\right)$ and the shortest $\tau_{\mathrm{av}}(1.42 \mathrm{~ns})$ among the three phases. These emission properties are analogous to those of the toluene solution (Fig. 8d): the polar excited state was weakly stabilized by the solvent. Therefore, the bluish-green emission from $1 \mathrm{G}$ could be derived from monomeric luminophores packed in a rigid crystalline phase. Although $\mathbf{1 G}^{\#}$ and $\mathbf{1 G}$ exhibited almost identical absorption spectra (Supplementary Fig. 4), $\mathbf{1 G}^{\#}$ had an emission maximum at $547 \mathrm{~nm}$, similar to that of $1 Y$ (Supplementary Fig. 6). Therefore, an energy transfer may occur from the excited state of the monomeric (1G) to the $\pi-\pi$ stacked chromophores (1Y) present in small quantities ${ }^{33}$.

Controlling crystallization using a lithium salt. The mechanically induced phase conversion from $\mathbf{1 0}$ to $\mathbf{1 G}^{\#}$ (that is, the less crystalline form of $\mathbf{1 G}$ ) was found to be caused by the nucleation and propagation of a crystalline phase in a mesomorphic state ${ }^{34}$. After an edge of the $\mathbf{1 0}$ sample was rubbed on a glass substrate, the crystallization developed toward the surrounding area at $\sim 1.0 \mathrm{~mm} \mathrm{~h}^{-1}$ at room temperature (panels a,b in Fig. 9A). The same phase transformation was triggered by touching a small 'seed' of $\mathbf{1 G}^{\#}$ to $\mathbf{1 0}$ (see Supplementary Movies 2 and 3), similar to crystal growth in solution. The phase conversion could be halted by heating the sample to $50^{\circ} \mathrm{C}$; this temperature is higher than the isotropization temperature $\left(T_{\text {iso }}\right)$ of $\mathbf{1 0}\left(47^{\circ} \mathrm{C}\right)$ but lower than that of $1 \mathrm{G}\left(54^{\circ} \mathrm{C}\right.$; panels $\left.\mathrm{c}, \mathrm{d}\right)$. The process was restarted when the sample was cooled to r.t. (panels e,f). Therefore, the phase conversion does not transmit to Iso. On the basis of these observations, we conclude that subjecting the metastable $\mathbf{1 0}$ to strong mechanical stimulation generates a small nucleus of thermodynamically stable $\mathbf{1 G}^{\#}$ that partially consists of $\pi-\pi$ stacked luminophores similar to $\mathbf{1 Y}$. The $\mathbf{1 G}^{\#}$ region could then spontaneously propagate. Thermal annealing of $\mathbf{1 G}^{\#}$ below its isotropization temperature $\left(T_{\text {iso }}=54{ }^{\circ} \mathrm{C}\right)$ induces another structural rearrangement to yield $\mathbf{1 G}$, freeing all of the luminophores from the $\pi-\pi$ stacking interactions.

Because of the 'seeding' effect of $\mathbf{1 G}^{\#}$ on $\mathbf{1 0}$, thermal writing created by a soldering iron (Fig. 9B) on the mechanically developed $\mathbf{1 G}^{\#}$ film $\left(>54^{\circ} \mathrm{C}\right.$ ) was automatically erased while the sample was maintained at r.t. for $1 \mathrm{~h}$ (the upper half of the characters 'CHIBA' in Fig. 9C). The volatility of the thermal writing on $\mathbf{1 G}^{\#}$ could be controlled by treating the substrate with aqueous solutions of a lithium salt. When the lower half of a sample containing the characters 'CHIBA' thermally written on $\mathbf{1 G}^{\#}$ was immersed into an aqueous $\mathrm{CF}_{3} \mathrm{SO}_{3} \mathrm{Li}$ solution $(\sim 10$ wt $\%)$ for a few seconds, the immersed portion of the characters continued to exhibit orange emission with almost the same fluorescence colours (the lower half of the characters 'CHIBA' in Fig. 9C). The PXRD analysis revealed that $\mathbf{1 0}$ was converted to $\mathbf{1 O}_{\mathbf{L i}}\left(T_{\text {iso }}=120^{\circ} \mathrm{C}\right)$ by forming chelation complexes in the TEG moieties (Supplementary Fig. 7) ${ }^{35,36}$. The interlayer distance was 


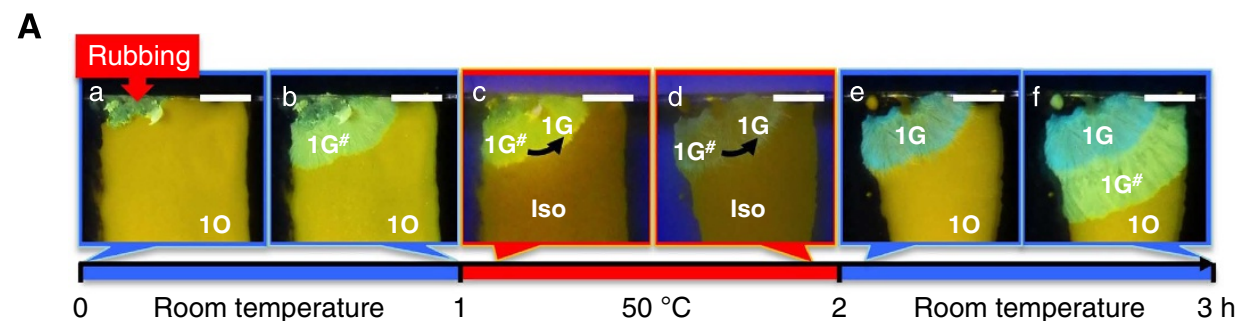

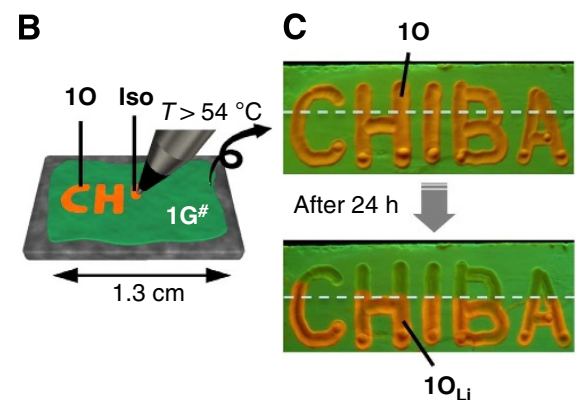

F Input substrate $\mathbf{G}$

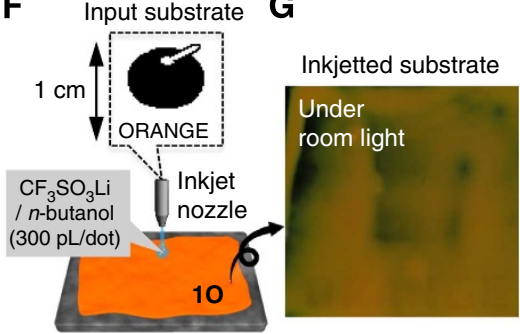

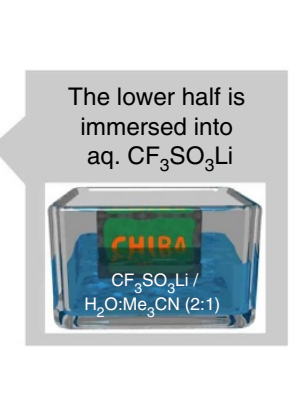

D

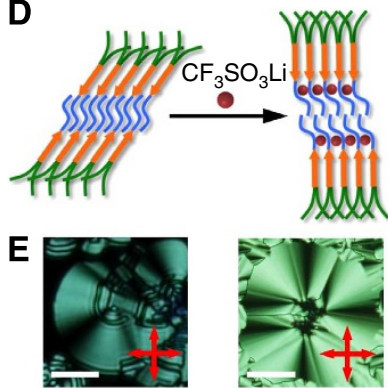

H

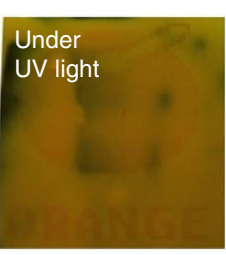

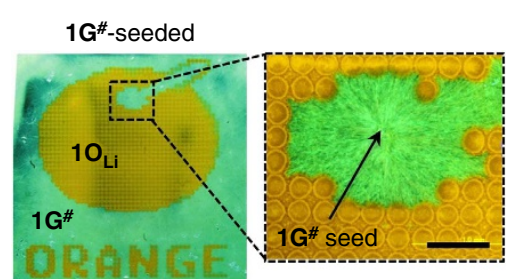

Figure 9 | Rubbing and seeding induced phase transition of $\mathbf{1 0}$ to 1G. (A) Photographs revealing the spontaneous propagation of $\mathbf{1 G}$ \# 10 under UV light. (a,b) Propagation at r.t. ; (c,d) propagation paused at $50^{\circ} \mathrm{C} ;(\mathrm{e}, \mathrm{f})$ propagation restarted at r.t. Scale bars, $1 \mathrm{~mm}$. (B) Schematic representation of thermal writing using Iso (converted to $\mathbf{1 0}$ upon cooling) generated with a soldering pen on $\mathbf{1 G}^{\#}$. (C) Photographs exhibiting the control over the auto-

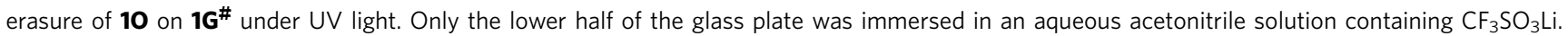
(D) Schematic representation of orientational change of $\mathbf{1}$ upon complexing with the lithium salt (shown by red balls). (E) POM images of $\mathbf{1 0}$ (left, scale bar, $10 \mu \mathrm{m}$ ) and $\mathbf{1 0}_{\mathbf{L i}}$ (right, scale bar, $50 \mu \mathrm{m}$ ). Red arrows indicate the direction of polarizer and analyser. (F) Schematic representation of inkjet writing of a cartoon picture of an orange fruit and the characters 'ORANGE' ( $\sim 1 \mathrm{~cm}$ in size) on $\mathbf{1 0}$ by using $\mathrm{n}$-butanol solution of $\mathrm{CF}_{3} \mathrm{SO}_{3} \mathrm{Li}(5 \mathrm{wt} \%)$. (G) Photographs of the inkjetted sample under room light (left) and under UV light (right). (H) Photograph of the inkjetted sample under UV light after $\mathbf{1 G}^{\#}$ seeding. Inset shows the fluorescent microscopic image of a part of the orange picture (scale bar, $0.5 \mathrm{~mm}$ ).

extended from $34.2 \AA$ in $\mathbf{1 O}$ to $60.1 \AA$ in $\mathbf{1 O}_{\mathrm{Li}}$; this change could be ascribed to the cancellation of the molecular tilt owing to the loss of the interdigitation of the TEG moieties upon chelation with the salt (Fig. 9D). The cancellation of the molecular tilt was further supported by the focal-conic fan texture of $\mathbf{1 O}_{\mathrm{Li}}$, which exhibited extinction brushes parallel to the polarizers (Fig. 9E). The inflexibility of the luminophore caused by the Li chelation and a less tilted structure in $\mathbf{1 O}_{\mathbf{L i}}$ are responsible for the higher $T_{\text {iso }}$ of $1 \mathbf{O}_{\mathbf{L i}}$.

The phase-transition property of 10 , which was controllable through the treatment with the lithium salt, was used in invisible inkjet imaging, which can be decoded by a 'molecular key' that can induce a selective phase change on 10 . We prepared thin films of $\mathbf{1 O}$ and created invisible images of a cartoon picture of an orange fruit and the characters 'ORANGE' on the film via inkjet printing ( $300 \mathrm{pL}$ per dot) with an $n$-butanol solution of $\mathrm{CF}_{3} \mathrm{SO}_{3} \mathrm{Li}$ (5 wt\%; Fig. 9F). The printed image was not clearly discernible under room light or even under UV illumination (Fig. 9G) because the resulting $1 \mathbf{O}_{\mathbf{L i}}$ has emission maximum identical to that of $\mathbf{1 0}$. However, when small crystallites of $\mathbf{1 G}^{\#}$ were seeded onto several parts of the film, only the background 10 was converted into $\mathbf{1 G}^{\#}$ after several hours. In contrast, the $\mathbf{1 O}_{\mathbf{L i}}$ in the imaged area remained unchanged, thereby revealing the printed images as orange emissions on the green-emitting background under UV illumination (Fig. 9H). Fluorescence microscopy observations revealed that the diffusion of the lithium salt on $\mathbf{1 0}$ was suppressed within $\sim 30 \mu \mathrm{m}$ (magnified image in Fig. 9H), which ensures a high resolution for imaging on the micrometre scale. Because the use of an aqueous solution of $\mathrm{CF}_{3} \mathrm{SO}_{3} \mathrm{Li}$ resulted in more unambiguous images (Supplementary Fig. 8), the selection of the solvent system for the inkjet procedure is important to minimize the diffusion of the salt within the mesophase.

\section{Discussion}

We have demonstrated that dipolar $\pi$-conjugated systems with proper amphiphilicity can form several metastable self-assembled phases with distinct packing states of the $\pi$-conjugated units and emission properties. The designed metastable state is converted into a more stable packing state via the application of both weak (1Y to $\mathbf{1 O}$ ) and strong (1O to $\mathbf{1 G}^{\#}$ ) mechanical stimuli (Fig. 1c). A mechanism for the mechano-induced phase conversion from $1 Y$ to $\mathbf{1 O}$ can be explained as follows: the evaporation of the acetonitrile solution containing $\mathbf{1}$ forms a metastable, multilamellar organized structure (1Y); this process is driven by the segregation of incompatible molecular components. The aggregation facilitates face-to-face $\pi-\pi$ stacking interactions between the dipolar $\pi$-conjugated systems to generate the yellow 
emission. Because of the energetically unfavourable parallel orientation of the dipole moments, a weak mechanical stimulus can easily convert this metastable, aggregated state into a mesomorphic state $(\mathbf{1 0})$. The similarity between the emission properties of $\mathbf{1 0}$ and the single crystal of $\mathbf{2}$ demonstrates that the dipolar $\pi$-conjugated systems are dynamically twisted in the mesomorphic state within the layers. The twisted $\pi$-conjugated system can induce intramolecular charge transfer, thus resulting in emission in the longest-wavelength region.

Although the dipolar repulsion between stacked $\pi$-conjugated systems in 1Y should be considerably dissolved by the transition into 10, it must still remain in this fluidal mesophase where $\pi$ conjugated systems can freely rotate within $\pi$-conjugated sublayers. Furthermore, the packing density mismatch between the $\pi$-conjugated sublayers and the interdigitated TEG sublayers as well as the Gemini-shaped aliphatic sublayers should make this phase thermodynamically unstable. This metastability facilitates crystallization to $\mathbf{1 G}^{\#}$ through mechanical stimulus or seeding to yield the green emission derived from the planar $\pi$-conjugated systems that are free of $\pi-\pi$ stacking interactions. The lithium salt adjusts the volume of the three incompatible molecular components of $\mathbf{1}$ by being sandwiched between the TEG chains of neighbouring parallel-oriented molecules, which stabilizes the mesophase against crystallization (Fig. 9D). We can apply this lithium-responsive property of $\mathbf{1 0}$ to invisible inkjet printing, which is subsequently revealed using a small crystal of the same material as a 'molecular key'.

In conclusion, we demonstrate that dipolar $\pi$-conjugated systems with proper amphiphilicity can form several metastable selfassembled phases with distinct molecular packing and emission properties. The designed metastable state is converted into a more stable packing state by the application of both weak and strong mechanical stimuli. The rationale for this behaviour involves the unfavourable parallel orientation of the molecular dipole moments that become kinetically trapped upon segregation between incompatible molecular regions. The present study provides a basic molecular strategy for the design of new mechano-responsive compounds with various $\pi$-conjugated luminophores.

\section{Methods}

Materials. Compounds $\mathbf{1}$ and $\mathbf{2}$ were synthesized according to Supplementary Fig. 9 based on the synthetic methods reported previously ${ }^{37}$. All starting materials and reagents were purchased from commercial suppliers and used as received. ${ }^{1} \mathrm{H}$ NMR (Supplementary Figs 10 and 12 ) and ${ }^{13} \mathrm{C}$ NMR (Supplementary Figs 11 and 13) and HRMS (Supplementary Figs 14 and 15) of 1 and 2 were included in the Supplementary Methods.
}

\footnotetext{
Measurements. The solvents for the spectroscopic measurements were all spectral grade and used without further purification. Solid-state UV/Vis absorption spectra were recorded on a JASCO V660 spectrophotometer. Fluorescence spectra were recorded on a JASCO FP6600 spectrofluorometer and a Hitachi F-7000 spectrometer. Fluorescence microscopic spectrum of the crystal of 2 was recorded on a photonic multichannel analyser (Hamamatsu Photonics). ${ }^{13} \mathrm{C}$ - and ${ }^{1} \mathrm{H}-\mathrm{NMR}$ spectra were recorded on Bruker DPS300, JEOL JNM-ECS400 and JEOL JNM-ECA500 spectrometers and chemical shifts are reported in p.p.m. $(\delta)$ with the signal of tetramethylsilane (TMS) as internal standard. electrospray ionization (ESI)- and atmospheric pressure chemical ionization-high-resolution mass spectrometry (ACPI-HRMS) were measured on a Exactive (Thermo Scientific). Elemental analyses were performed at Chemical Analysis Center in Chiba University. Fluorescence quantum yields were measured on Absolute PL Quantum Yield Measurement System C9920-02 (Hamamatsu Photonics). Emission lifetime measurements were recorded on Quantaurus-Tau spectrometers (Hamamatsu Photonics). DSC was performed on SII DSC6220. Heating and cooling rate is $2{ }^{\circ} \mathrm{Cmin}^{-1}$. Polarized OM observation was carried out using an Olympus BX51 OM system with a Linkam temperature-controlled heating stage. SEM observation was acquired by using JEOL JSM-6330F SEM. AFM observation was carried out using Nanoscope Multimode 8 (Bruker AXS). PXRD analysis was carried out with a Rigaku Rint-2200 X-ray diffractometer with monochromated $\mathrm{CuK} \alpha_{1}(\lambda=1.54 \AA)$ radiation and temperature-controlled heating stage. $2 \mathrm{D}$ WAXD measurements were performed with synchrotron radiation $(\lambda=0.75 \AA)$ at BL02B2 in SPring- 8
}

with the approval of Japan Synchrotron Radiation Research Institute (JASRI). The samples were prepared on a glass substrate (thickness $=0.03 \mathrm{~mm}$ ).

In-plane X-ray diffraction. In-plane X-ray diffraction of sheared 1G film was carried out with TRY-DXGSA-HHIP vertical X-ray diffractometer (TRY SE Co., Ltd) equipped with an imaging plate holder and a hot stage parallel to the glass plate. The sample sheared on a glass plate (glass thickness: $0.05 \mathrm{~mm}$ ) was irradiated by monochromated $\mathrm{CuK} \alpha_{1} \mathrm{X}$-ray $(\lambda=1.54 \AA$, beam diameter: $0.5 \mathrm{~mm}$; voltage: $40 \mathrm{kV}$; current: $30 \mathrm{~mA}$; and camera length: $138 \mathrm{~mm}$ ). The diffraction was recorded on an imaging plate $(200 \mathrm{~mm} \times 200 \mathrm{~mm})$, and the image was read by an FLA-7000 imaging plate reader (Fujifilm Co., Ltd).

Single-crystal X-ray analysis. Measurements of the single crystal of $\mathbf{2}$ were performed on a Rigaku R-AXIS RAPID diffractometer equipped with a graphitemonochromated Mo-K $\alpha$ radiation source. The diffraction data were collected at various temperatures and the crystallographic parameters are summarized in Supplementary Table 3 . The structure was determined using direct methods and was expanded using Fourier techniques. Non-hydrogen atoms were refined anisotropically, except in the case of the disordered oligo( $p$-phenylenevinylene) cores. Hydrogen atoms were refined using the riding model. All the calculations were performed using the CrystalStructure crystallographic software package, except for refinement, which was performed using the SHELXL-97 software programme.

Inkjet printing. Inkjet printing experiments were performed using a LaboJet-500 inkjet printer, Microjet Corporation, Japan. An $n$-butanol solution of $\mathrm{CF}_{3} \mathrm{SO}_{3} \mathrm{Li}$ (5 wt $\%$ ) was dotted ( $300 \mathrm{pL}$ per dot) onto thin films of $\mathbf{1 0}$ to draw invisible images. After the inkjet printing, small crystallites of $\mathbf{1 G}^{\#}$ were seeded onto the film. After the sample was maintained at r.t. for $3 \mathrm{~h}$, the background $\mathbf{1 0}$ was fully converted to $\mathbf{1 G}^{\#}$ to reveal the invisible images.

\section{References}

1. Srinivasan, S., Babu, P. A., Mahesh, S. \& Ajayaghosh, A. Reversible selfassembly of entrapped fluorescent gelators in polymerized styrene gel matrix: Erasable thermal imaging via recreation of supramolecular architectures. J. Am. Chem. Soc. 131, 15122-15123 (2009).

2. Mutai, T., Satou, H. \& Araki, K. Reproducible on-off switching of solid-state luminescence by controlling molecular packing through heat-mode interconversion. Nat. Mater. 4, 685-687 (2005).

3. Irie, M., Fukaminato, T., Sasaki, T., Tamai, N. \& Kawai, T. Organic chemistry: A digital fluorescent molecular photoswitch. Nature 420, 759-760 (2002).

4. Kishimura, A., Yamashita, T., Yamaguchi, K. \& Aida, T. Rewritable phosphorescent paper by the control of competing kinetic and thermodynamic self-assembling events. Nat. Mater. 4, 546-549 (2005).

5. Sagara, Y. \& Kato, T. Mechanically induced luminescence changes in molecular assemblies. Nat. Chem. 1, 605-610 (2009).

6. Balch, A. L. Dynamic crystals: visually detected mechanochemical changes in the luminescence of gold and other transition-metal complexes. Angew. Chem. Int. Ed. Engl. 48, 2641-2644 (2009).

7. Sagara, Y., Yamane, S., Mutai, T., Araki, K. \& Kato, T. A Stimuli-responsive, photoluminescent, anthracene-based liquid crystal: emission color determined by thermal and mechanical processes. Adv. Funct. Mater. 19, 1869-1875 (2009).

8. Chi, Z. et al. Recent advances in organic mechanofluorochromic materials. Chem. Soc. Rev. 41, 3878-3896 (2012).

9. Ito, $\mathrm{H}$. et al. Reversible mechanochromic luminescence of $\left[\left(\mathrm{C}_{6} \mathrm{~F}_{5} \mathrm{Au}\right)_{2}(\mathrm{~m}-1,4-\right.$ diisocyanobenzene)]. J. Am. Chem. Soc. 130, 10044-10045 (2008).

10. Yoon, S.-J. et al. Multistimuli two-color luminescence switching via different slip-stacking of highly fluorescent molecular sheets. J. Am. Chem. Soc. 132, 13675-13683 (2010).

11. Sagara, Y., Mutai, T., Yoshikawa, I. \& Araki, K. Material design for piezochromic luminescence: hydrogen-bond-directed assemblies of a pyrene derivative. J. Am. Chem. Soc. 129, 1520-1521 (2007).

12. Luo, X. et al. Reversible switching of the emission of diphenyldibenzofulvenes by thermal and mechanical stimuli. Adv. Mater. 23, 3261-3265 (2011).

13. Yuan, W. Z. et al. Synergy between twisted conformation and effective intermolecular interactions: strategy for efficient mechanochromic luminogens with high contrast. Adv. Mater. 25, 2837-2843 (2013).

14. Shan, G.-G. et al. Controllable synthesis of iridium(III)-based aggregationinduced emission and/or piezochromic luminescence phosphors by simply adjusting the substitution on ancillary ligands. J. Mater. Chem. C 1, 1440-1449 (2013).

15. Wang, J. et al. Click synthesis, aggregation-induced emission, E/Z isomerization, self-organization, and multiple chromisms of pure stereoisomers of a tetraphenylethene-cored luminogen. J. Am. Chem. Soc. 134, 9956-9966 (2012). 
16. Zhao, N. et al. Benzothiazolium-functionalized tetraphenylethene: an AIE luminogen with tunable solid-state emission. Chem. Commun. 48, 8637-8639 (2012).

17. Lowe, C. \& Weder, C. Oligo(p-phenylene vinylene) excimers as molecular probes: deformation-induced color changes in photoluminescent polymer blends. Adv. Mater. 14, 1625-1629 (2002).

18. Sagara, Y. \& Kato, T. Stimuli-responsive luminescent liquid crystals: change of photoluminescent colors triggered by a shear-induced phase transition. Angew. Chem. Int. Ed. Engl. 47, 5175-5178 (2008).

19. Sagara, Y. \& Kato, T. Brightly tricolored mechanochromic luminescence from a single-luminophore liquid crystal: reversible writing and erasing of images. Angew. Chem. Int. Ed. Engl. 50, 9128-9132 (2011).

20. Teng, M.-J., Jia, X.-R., Chen, X.-F. \& Wei, Y. A Dipeptide-based multicoloredswitching luminescent solid material: When molecular assemblies meet mechanochemical reaction. Angew. Chem. Int. Ed. Engl. 51, 6398-6401 (2012).

21. Perruchas, S. et al. Mechanochromic and thermochromic luminescence of a copper iodide cluster. J. Am. Chem. Soc. 132, 10967-10969 (2010).

22. Yeh, M.-C. et al. Amphiphilic design of a discotic liquid-crystalline molecule for dipole manipulation: hierarchical columnar assemblies with a $2 \mathrm{D}$ superlattice structure. Angew. Chem. Int. Ed. Engl. 52, 1031-1034 (2013).

23. Li, W.-S. et al. Amphiphilic molecular design as a rational strategy for tailoring bicontinuous electron donor and acceptor arrays: Photoconductive liquid crystalline oligothiophene-C60 dyads. J. Am. Chem. Soc. 130, 8886-8887 (2008).

24. Sakurai, T. et al. Prominent electron transport property observed for triply fused metalloporphyrin dimer: directed columnar liquid crystalline assembly by amphiphilic molecular design. J. Am. Chem. Soc. 130, 13812-13813 (2008)

25. Hulvat, J. F., Sofos, M., Tajima, K. \& Stupp, S. I. Self-assembly and luminescence of oligo(p-phenylene vinylene) amphiphiles. J. Am. Chem. Soc 127, 366-372 (2005).

26. Hoeben, F. J. M. et al. Direct visualization of efficient energy transfer in single oligo(p-phenylene vinylene) vesicles. Angew. Chem. Int. Ed. Engl. 45, 1232-1236 (2006)

27. Ajayaghosh, A. \& Praveen, V. K. p-Organogels of self-assembled p-phenylenevinylenes: soft materials with distinct size, shape, and functions. Acc. Chem. Res. 40, 644-656 (2007).

28. Harada, J. \& Ogawa, K. X-ray diffraction analysis of nonequilibrium states in crystals: observation of an unstable conformer in flash-cooled crystals. J. Am. Chem. Soc. 126, 3539-3544 (2004).

29. Kasha, M., Rawls, H. R. \& El-Bayoumi, M. A. Exciton model in molecular spectroscopy. Pure Appl. Chem. 11, 371-392 (1965).

30. Yagai, S. et al. Diversification of self-organized architectures in supramolecular dye assemblies. J. Am. Chem. Soc. 129, 13277-13287 (2007).

31. Yagai, S., Higashi, M., Karatsu, T. \& Kitamura, A. Tunable interchromophore electronic interaction of a merocyanine dye in hydrogen-bonded supramolecular assemblies scaffolded by bismelamine receptors. Chem. Commun. 1500-1502 (2006).

32. Rosch, U., Yao, S., Wortmann, R. \& Wurthner, F. Fluorescent H-aggregates of merocyanine dyes. Angew. Chem. Int. Ed. Engl. 45, 7026-7030 (2006).
33. Peng, K.-Y., Chen, S.-A. \& Fann, W.-S. Efficient light harvesting by sequential energy transfer across aggregates in polymers of finite conjugational segments with short aliphatic linkages. J. Am. Chem. Soc. 123, 11388-11397 (2001).

34. Ito, H. et al. Mechanical stimulation and solid seeding trigger single-crystal-tosingle-crystal molecular domino transformations. Nat. Commun. 4, 2009 (2013).

35. Percec, V. et al. Self-assembly of taper-shaped monoesters of oligo(ethylene oxide) with 3,4,5-tris(p-dodecyloxybenzyloxy)benzoic acid and of their polymethacrylates into tubular supramolecular architectures displaying a columnar mesophase. J. Chem. Soc. Perkin Trans. 1, 2799-2811 (1993).

36. Lee, M., Oh, N.-K., Lee, H.-K. \& Zin, W.-C. Liquid crystalline assembly of a diblock rod-coil polymer based on poly(ethylene oxide) and its complexes with $\mathrm{LiCF}_{3} \mathrm{SO}_{3}$. Macromolecules 29, 5567-5573 (1996).

37. Yagai, S. et al. Supramolecularly engineered aggregation of a dipolar dye: vesicular and ribbonlike architectures. Angew. Chem. Int. Ed. 49, 9990-9994 (2010).

\section{Acknowledgements}

This work was supported partially by KAKENHI (24685022) from the MEXT. We acknowledge Y. Hirai and M. Suzuki for their help in taking photographs.

\section{Author contributions}

S.Y., S.O. and Y.N. conceived and designed the projects; S.O. and Y.N. synthesized the compounds and contributed on most of the experimental work; D.K. and H.Y. measured fluorescence quantum yield; M.Y. performed the AFM experiments; T.S. and H.I. performed the single-crystal X-ray analysis and fluorescence lifetime measurements; K.K. helped the discussion on the PXRD analysis; S.Y. and H.I. wrote the manuscript; T.K. and A.K. discussed the results and commented on the manuscript.

\section{Additional information}

Accession codes: The X-ray crystallographic coordinate for 2 reported in this article have been deposited at the Cambridge Crystallographic Data Centre (CCDC) under deposition numbers CCDC 986425 . These data can be obtained free of charge from The Cambridge Crystallographic Data Centre via www.ccdc.cam.ac.uk/data_request/cif.

Supplementary Information accompanies this paper at http://www.nature.com/ naturecommunications

Competing financial interests: The authors declare no competing financial interests.

Reprints and permission information is available online at http://npg.nature.com/ reprintsandpermissions/

How to cite this article: Yagai, S. et al. Design amphiphilic dipolar $\pi$-systems for stimuli-responsive luminescent materials using metastable states. Nat. Commun. 5:4013 doi: 10.1038/ncomms5013 (2014). 